\title{
CONCEPCIONES ALTERNATIVAS Y DIFICULTADES DE APRENDIZAJE EN ELECTROSTÁTICA. SELECCIÓN DE CUESTIONES ELABORADAS PARA SU DETECCIÓN Y TRATAMIENTO
}

\author{
FURIÓ, CARLES ${ }^{1}$ y GUISASOLA, JENARO ${ }^{2}$ \\ 1 Departamento de Didáctica de la Ciencias Experimentales. Universidad de Valencia. \\ 2 Departamento de Física Aplicada I. Universidad del País Vasco.
}

\begin{abstract}
SUMMARY
The work we are presenting here tries to summarise the contributions made by research on the ideas and difficulties of students within the field of Electrostatics. These researches reveal that the concepts introduced in this domain, such as electric field and potential, are necessary prerequisites so as to acquire a scientific vision of electromagnetic phenomena. Thus, taking into account the importance of the concepts involved for a scientific vision of the circuits of continuous current and, in general, of the electromagnetic theory, we think it is important for teachers to have a first idea on which are the most problematic conceptions or at least, those that have been studied most.
\end{abstract}

\section{INTRODUCCIÓN}

La enseñanza de los conocimientos teóricos es un problema que preocupa cada vez más al profesorado debido a la constatación de altos porcentajes de respuestas erróneas de los estudiantes a cuestiones teóricas que exigen no sólo la mera repetición de la teoría impartida en clase sino la aplicación creativa de dichos conocimientos. Fueron estas nuevas formas de preguntar -no las de repetición rutinaria- las que llevaron a la detección de respuestas erróneas, ya que estas «preguntas inteligentes» son las que obligan a una aplicación significativa de los conocimientos aprendidos.

El estudio de las respuestas de los estudiantes a este tipo de preguntas ha dado lugar a una de las líneas de investigación en didáctica de las ciencias que más se ha desarrollado en los últimos veinte años, la que se ha centrado en el estudio de las concepciones alternativas de los estudiantes (Wandersee, et al., 1994) y que muestran la existencia de verdaderas dificultades de aprendizaje en diferentes campos básicos de la física (Viennot, 1985; Driver, 1989). La hipótesis de trabajo actualmente aceptada explicita que los estudiantes poseen ideas antes y durante el proceso de aprendizaje que influyen decisivamente en el mismo (Ausubel, 1978).

Una primera cuestión que llama la atención en estos trabajos es la diversidad terminológica usada para nombrar las ideas distintas a las científicas que tiene el alumnado. Hay autores como Abimbola (1988) que han registrado hasta veintiocho términos diferentes para nombrarlas: ideas erróneas o errores conceptuales, concepciones precientíficas o preconcepciones, razonamiento de sentido común, modelos personales de la realidad, ciencia de los niños... Estas diferencias terminológicas 
se derivan del mayor o menor énfasis puesto por los investigadores en los «errores de los estudiantes» o en «cómo son sus ideas». Cuando el investigador concede mayor importancia al contenido que se va a enseñar y menos al conocimiento personal, centrará su atención en los defectos que tiene el alumnado y utilizará el descriptor error conceptual. Si, por el contrario, le interesa más conocer las ideas con que el alumnado interpreta el mundo y las considera válidas y coherentes -aunque sean diferentes a las científicas-, entonces será propenso a utilizar términos como concepciones, constructos personales... (Furió, 1996).

A pesar de esta gran disparidad terminológica hay un cierto consenso entre los investigadores que trabajan en este campo de estudios a usar la expresión concepciones alternativas (Wandersee, et al., 1994). De ahí que la mayor parte se integre en lo que se denomina movimiento de las concepciones alternativas. Esta línea de investigación es, de facto, la más importante de las existentes en la didáctica de las ciencias. Así, en la revista Enseñanza de las Ciencias aproximadamente el $38 \%$ del total de artículos publicados en el período 1983-92 se ha dedicado específicamente al estudio de las dificultades de los estudiantes sobre conceptos científicos (Moreira, 1994). En el ámbito internacional, en la última de las revisiones bibliográficas realizadas por Pfundt y Duit sobre concepciones alternativas (1994), los artículos referenciados son más de 3.000. Ahora bien, la distribución de este enorme volumen de producción no ha sido uniforme en todas las disciplinas, siendo el $61 \%$ del total sobre conceptos de física, el 20\% de biología, el 18\% de química y el $1 \%$ de geología. Y dentro de la física los conceptos de mecánica (tales como las fuerzas, el movimiento, la aceleración o la gravedad) han sido los más estudiados (Carrascosa y Gil, 1992). Le siguen en orden decreciente los de electricidad, calor y temperatura $(6 \%)$ (Cervantes, 1987), energía (6\%) (Hierrezuelo, 1986), óptica (6\%) (Perales y Nievas, 1988) y física moderna (1\%).

Respecto del campo de la electricidad, que es el tema que nos ocupa en este trabajo, hay que indicar que han sido estudiadas ampliamente las ideas de los estudiantes en el dominio de la electrocinética y, en particular, en los circuitos eléctricos de corriente continua (Closset, 1983; Dupin y Joshua, 1986; Shipstone et al., 1988; Manrique et al., 1989). Estos estudios muestran que, incluso después de un largo período de instrucción, los estudiantes presentan confusiones al tratar de establecer la causa del cambio de movimiento de las cargas y su relación con las magnitudes físicas que se introducen (intensidad de corriente, diferencia de potencial, resistencia) en la electrocinética.

Las dificultades encontradas en los trabajos mencionados revelan que los conceptos implicados son de alta demanda cognitiva y que además se apoyan en prerrequisitos fundamentales que son introducidos en la electrostática como campo y potencial eléctricos. En este sentido, recientes publicaciones (Chabay y Sherwood, 1995) indican que una clara comprensión de los conceptos introducidos en electrostática es esencial si uno

quiere adquirir una visión científica de los fenómenos electromagnéticos. Así pues, el trabajo que aquí presentamos trata de resumir las aportaciones que han realizado las investigaciones sobre las ideas de los estudiantes y sus dificultades en el dominio de la electrostática. Estas investigaciones son recientes (comienzan en la década de los noventa) y no son muy numerosas, pero, teniendo en cuenta la importancia de los conceptos implicados para una visión científica de los circuitos de corriente continua y, en general, de la teoría electromagnética, creemos importante que el profesorado pueda obtener una primera idea de cuáles son las concepciones más problemáticas o, al menos, las más estudiadas.

Hemos intentado reagrupar las cuestiones de los diversos trabajos en una serie de epígrafes que hacen referencia a diferentes aspectos dentro de la electrostática. Sin embargo, es necesario tener presente que la mayor parte de las veces se trata de aspectos relacionados; por lo que no se deberá perder su visión de conjunto. No obstante, hemos preferido realizar una clasificación de las cuestiones (a veces no exenta de cierta arbitrariedad) a una presentación desestructurada del conjunto de las mismas, que podría hacer pensar que las concepciones alternativas son ideas sueltas, en contra de lo establecido por diferentes investigaciones, que han mostrado que estas ideas están dotadas de cierta coherencia interna, estructurándose en auténticos «marcos conceptuales alternativos» (Gianneto et al., 1992).

Cada cuestión se presenta con su enunciado y su correspondiente referencia (artículo o libro donde es posible localizarla). Después se exponen los comentarios de la cuestión junto con algunos resultados de su aplicación.

\section{DIFICULTADES DE LOS ESTUDIANTES PARA INTERPRETAR FENOMENOS ELEC- TROSTÁTICOS BÁSICOS COMO INTERAC- CIONES ENTRE CARGAS PUNTUALES, FENOMENOS TRIBOELECTRICOS O DE IN- DUCCION ELECTRICA}

En este apartado hacemos referencia a algunas cuestiones que tienen como objetivo investigar hasta qué punto los estudiantes poseen una comprensión integrada de los fenómenos eléctricos.

1.1. Dos bolas cargadas de poliestireno tienen la misma masa $m$ y están suspendidas de un hilo, una al lado de la otra. La carga de una de las bolas es doble que la de la otra. Elegir el diagrama apropiado para mostrar el desplazamiento angular relativo de las bolas.

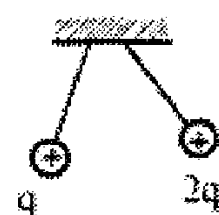

3

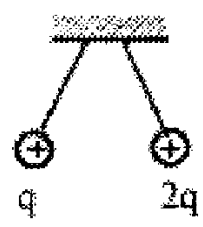

(b)

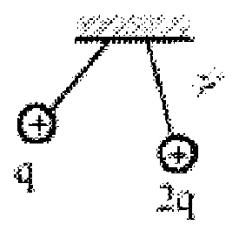

(s) 


\section{Referencia: Galili (1995)}

Comentario: Para contestar correctamente la cuestión planteada no es necesario un nivel teórico alto en electricidad. Al contrario, con conocer que las cargas de igual signo se repelen y las leyes de Newton impartidas en mecánica es suficiente. Así, una aplicación correcta de la tercera ley de Newton nos indica que el desplazamiento angular debe ser simétrico para las dos cargas. Sin embargo, alrededor de un tercio de los estudiantes encuestados ( 88 alumnos de niveles similares a $3^{\circ}$ de BUP, COU y curso de aptitud pedagógica) toma las opciones incorrectas $(a$ y $c)$, otro tercio elige la respuesta correcta (b) y el resto no responde. Por tanto, dos tercios de los estudiantes no aplican correctamente la tercera ley de Newton para una interacción electrostática entre dos cargas puntuales. Parece que los estudiantes consideran de forma errónea la cuestión de que «a mayor carga, mayor fuerza» y esto hace que se viole el principio de acción y reacción (la simetría de la fuerza de interacción), que ha sido reiteradamente impartido en mecánica al estudiar las leyes de Newton. El resultado sugiere que los estudiantes no tienen en cuenta lo aprendido en otras partes de la física (en este caso, la mecánica) para analizar cuestiones de electricidad y que las dificultades de aprendizaje detectadas en mecánica se extienden a otros campo de la física como el electromagnetismo, donde se aplican conceptos anteriores como fuerza, interacciones, transformaciones energía-trabajo...

1.2. Sea un sistema formado por dos cargas puntuales, una de las cargas se rodea de una esfera metálica no cargada y aislada, tal y como se ve en la figura. ¿Qué fuerza actúa sobre cada carga?
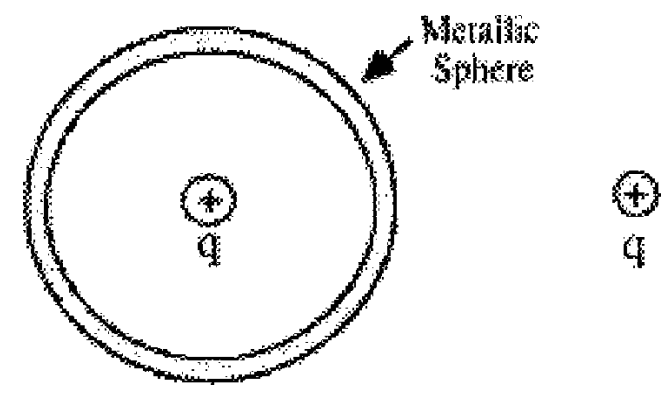

\section{Referencia: Galili (1995)}

Comentario: Esta cuestión de nuevo trata sobre la interacción entre dos cargas puntuales en situación electrostática «no simétrica» (una de ellas «encerrada» dentro de una corteza esférica metálica). Los resultados muestran que las cuestión está lejos de ser sencilla o de tener una única interpretación para los estudiantes. El 68\% de las respuestas analiza el fenómeno como si la situación fuera simétrica (interacción directa entre las dos cargas). Dentro de estas respuestas, el 33\% ignora la presencia de la corteza esférica, el $25 \%$ indica que no hay interacción y el $10 \%$ explica que la interacción es igual en ambas cargas pero más pequeña que si no hubiera corteza.

Estos resultados sugieren que los estudiantes, a la hora de explicar la interacción entre las cargas, saben que la acción en una carga implica la reacción en la otra, pero si profundizamos un poco, vemos que ese análisis es demasiado simple. En efecto, los estudiantes en sus explicaciones no tienen en cuenta (sólo un 1\% de respuestas correctas) el efecto de polarización de la esfera. La respuesta correcta al problema planteado considera que se ejerce fuerza sobre la carga externa mientras que la fuerza ejercida sobre la carga interna es nula. Ello no significa que no se cumple el principio de acción y reacción, pero considerando la interacción entre la carga externa y las inducidas en la esfera metálica (Fig. 1). Así pues, los resultados de esta cuestión sugieren que los estudiantes no sólo hacen una aplicación superficial de la tercera ley de Newton (dificultad ya observada en los trabajos sobre concepciones alternativas en el campo de la mecánica), sino que presentan serias dificultades a la hora de analizar el comportamiento de un metal cuando hay interacciones eléctricas.

Figura 1

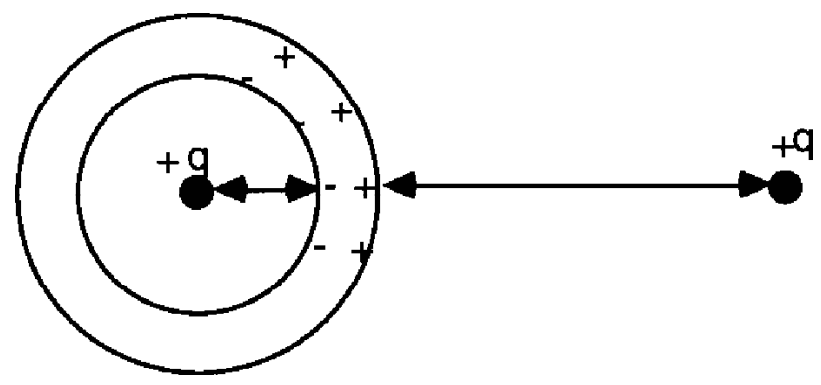

1.3. Al frotar con un paño de lana una barra metálica, se comprueba que ésta no queda electrizada. ¿Cómo se explica que no se electrice la barra?

Referencia: Furió y Guisasola (1993)

Comentario: Aunque el fenómeno planteado en la cuestión es en apariencia muy sencillo y es familiar a la experiencia de los estudiantes, el alto fracaso registrado nos indica que éstos tienen serias dificultades en su interpretación. En efecto, la cuestión fue contestada por estudiantes de bachillerato y primer ciclo de universidad, siendo alrededor del $22 \%$ las respuestas correctas. Así, la respuesta correcta necesita argumentos que se pueden articular en tres etapas: $a$ ) admitir la existencia previa de cargas positivas y negativas en la barra de metal antes de que se frote; $b$ ) admitir que el frotamiento implica a nivel microscópico el intercambio de cargas entre cuerpos frotados; $c$ ) tener en cuenta que, al no estar la barra de metal aislada (si bien no se indica a propósito en el enunciado), se produce una conducción de cargas a tierra. Los resultados indican que los estudiantes no 
tienen en cuenta la naturaleza eléctrica de los metales a la hora de analizar el fenómeno presentado, lo que vendría a confirmar las conclusiones de otras investigaciones como la presentada en el apartado 1.2. Así mismo, los bajos resultados obtenidos (no hay mejora a pesar de aumentar el nivel educativo) pueden explicarse también teniendo en cuenta dificultades metodológicas como la utilización de un causalismo simple en el análisis de la cuestión (p.e., «no se electriza porque la barra es de metal», «es necesario frotar más», «la estructura del metal no deja escapar electrones al paño»...). Esta forma de razonamiento es característica de la metodología de «sentido común», en la cual se buscan argumentos directos y rápidos que expliquen el fenómeno (Gil y Carrascosa, 1985).

1.4. Se aproxima una hoja de plástico cargada al extremo de una barra larga de madera sin tocarla, como se ve en la figura. En el otro extremo de la barra hay una bolita de poliuretano. Explica si será atraída, o no, la bolita.

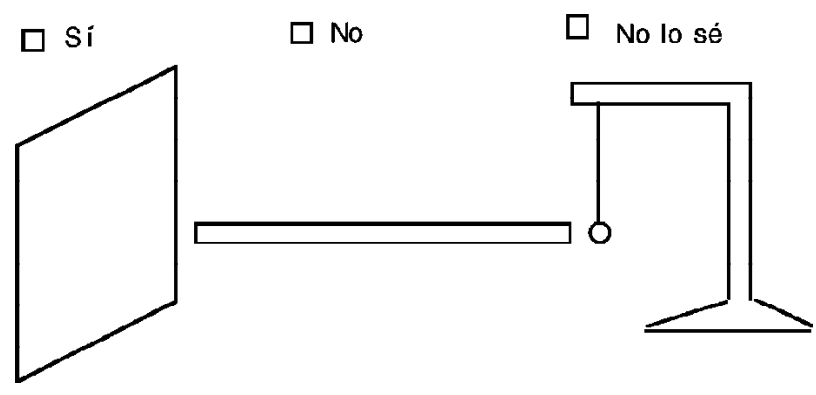

Referencia: Guisasola y Furió (1994)

Comentario: En la interpretación del fenómeno de inducción presentado es necesario tener en cuenta la polarización del medio (la barra de madera) y la transmisión de la interacción eléctrica a través del mismo. Sin embargo, la mayoría de los estudiantes de bachillerato (más de las tres cuartas partes de las respuestas) y primer ciclo de universidad (más de la mitad de las respuestas) encuestados indica erróneamente que la bolita no es atraída. La explicación más frecuente que dan los estudiantes se basa en considerar que la carga eléctrica no puede «fluir» a través de la madera al ser ésta aislante y sugiere que la mayoría de estudiantes encuestados utiliza un modelo de fluido eléctrico para la carga al analizar fenómenos de interacción eléctrica entre un cuerpo cargado y otro neutro. Esta interpretación se aleja mucho del modelo submicroscópico de la naturaleza eléctrica de la materia actualmente aceptado. En efecto, consideran que la naturaleza «no eléctrica» de la madera (aislante) no deja pasar la carga al extremo enfrentado con el péndulo y que no es posible la interacción eléctrica (debido a razones de proximidad entre cargas).

1.5. Un bolígrafo frotado es capaz de atraer un trocito de papel próximo a él. Explica cómo se produce esta interacción.
Referencia: Furió y Guisasola (1998a)

Comentario: Cuando los estudiantes tratan de analizar fenómenos de inducción o de influencia local que les son familiares, se constata que existen dificultades dado el bajo índice de respuestas correctas. La explicación correcta del fenómeno va aumentando lentamente con la instrucción ( $13 \%$ en $3^{\circ}$ de BUP, $27 \%$ en COU, $30 \%$ en $1^{\circ}$ de físicas y $31 \%$ en $3^{\circ}$ de físicas). Ahora bien, entre las posibles explicaciones correctas llama la atención la casi nula utilización del concepto de campo eléctrico (porcentajes muy bajos) que hacen alumnos universitarios con varios años de instrucción en la materia. También se constata una diversificación en los argumentos, algunos de los cuales recuerdan interpretaciones surgidas a lo largo de la historia de la electricidad y que son anteriores a las del modelo coulombiano. Así, un tipo de respuestas detectada es una primera concepción alternativa que presenta cierta similitud con el modelo de Gilbert, en el que se habla del efecto «halo» producido por los cuerpos cargados para explicar la atracción que ejercen sobre cuerpos ligeros situados en su proximidad ( $38 \%$ en bachillerato, $23 \%$ en $1^{\circ}$ de físicas y $17 \%$ en $3^{\circ}$ de físicas). Otro tipo de respuestas (29\% en $3^{\circ}$ de BUP, $22 \%$ en COU, $25 \%$ en $1^{\circ}$ de físicas y $19 \%$ en $3^{\circ}$ de físicas) representa un segundo modelo alternativo en el que se justifica la atracción de los cuerpos cargados en base a la existencia de cuerpos cargados de signo opuesto. Así, se considera que de alguna forma los trozos de papel están cargados con signo opuesto a la pajita frotada. En este caso, las explicaciones de los estudiantes invertirían la relación causa-efecto. Es decir, los cuerpos se atraen y, por tanto, están cargados con cargas de distinto signo, lo que implica afirmar erróneamente que los trozos de papel están cargados. Por el contrario, lo que la teoría expone es que, cuando hay cuerpos cargados con distinto signo, se produce una atracción.

\section{DIFICULTADES DE APRENDIZAJE DEL CONCEPTO DE CAMPO ELECTRICO}

2.1. Un péndulo electrostático cargado positivamente es atraído por una barra de plástico cargada negativamente y es repelido por una barra de plástico cargada positivamente. ¿Cómo explicarías el fenómeno?

\section{Referencia: Nardi y Carvalho (1990)}

Comentario: Esta pregunta se realiza dentro de un estudio psicogenético sobre las ideas que influyen en el concepto de campo. Se realizaron 45 entrevistas a estudiantes de 6 a 17 años teniendo como base cuatro experiencias, una de las cuales es la indicada. En este estudio se demuestra que las respuestas de los estudiantes se pueden clasificar en tres niveles: $a$ ) estudiantes que no conciben la acción a distancia y no logran relacionar el experimento con una única causa; $b$ ) estudiantes que atribuyen la acción a la existencia de campo en todos los puntos en torno a una fuente generadora, que creen la 
fuerza ejercida depende de la distancia y que el campo se representa por una magnitud vectorial con dirección y sentido; y $c$ ) estudiantes que reconocen todas las propiedades de un campo físico en diferentes contextos (eléctrico, gravitatorio...) y, además, utilizan un lenguaje científico coincidente con la teoría enseñada en el bachillerato. Esta clasificación por niveles del avance en el aprendizaje de concepto de campo sugiere a los autores un cierto paralelismo con la historia del desarrollo del concepto a lo largo de la teoría electromagnética, y ello lleva a proponer la utilización de la historia de la ciencia para detectar los obstáculos epistemológicos y poder facilitar a los estudiantes el paso de un nivel a otro de dicho concepto.

2.2. Dibujar el campo creado por una carga $Q=+2 \mathrm{C}$ en el punto A en los tres casos siguientes :

a) En A hay una carga $q=+1 \mathrm{C}$.

b) En A hay una carga $q=-1 \mathrm{C}$.

c) En A no hay nada.

Referencia: Solbes y Martín (1991), Guisasola (1996)

Comentario: Esta pregunta ha sido aplicada por Solbes y Martín (1986) a una muestra de 135 estudiantes de $2^{\circ}$ de BUP y 131 de COU. Los resultados $\left(5 \%\right.$ en $2^{\circ}$ BUP y $2 \%$ en COU de respuestas correctas) sugieren que la gran mayoría de los estudiantes identifica el vector intensidad de campo eléctrico con la fuerza existente entre ambas cargas y por ello la mayoría contesta en el caso $c$ que no hay campo. Los resultados muestran que la mayoría de los estudiantes es incapaz de dar un significado físico al campo independientemente de la fuerza.

2.3. Sean dos cargas Q y Q' ambas positivas, Q'> Q, situadas en dos puntos A y B separados una distancia $r$.

a) ¿El campo E creado por $\mathrm{Q}$ en $\mathrm{B}$ es mayor, menor o igual que el campo E' creado por Q' en A?

b) La fuerza que Q produce sobre Q' es mayor, menor o igual que la fuerza Q' sobre Q?

\section{Referencia: Solbes y Martín (1991)}

Comentario: Esta pregunta se aplicó a la misma muestra que la cuestión anterior y se obtuvo que la respondían correctamente el $26 \%$ de $2^{\circ}$ BUP y el $31 \%$ de COU. El resultado confirma la conclusión de la pregunta anterior en el sentido de que el concepto de campo no se distingue claramente del de fuerza. Así, el campo sirve para calcular la fuerza, por lo tanto, es un mero artificio, un lenguaje redundante, incluso más complicado que el anterior y así no se comprende su necesidad ni su significado.

2.4. La figura es una representación gráfica de un campo eléctrico. Una carga puntual negativa es colocada en el punto $a$ sin velocidad inicial. Explicar y dibujar la trayectoria del movimiento de esta partícula negativa.

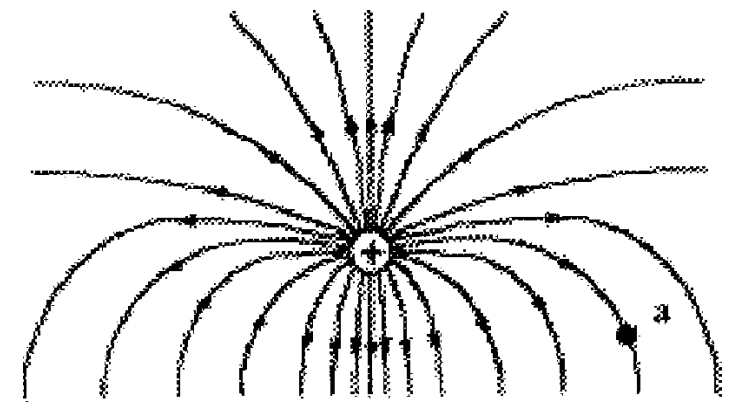

Referencia: Galili (1995)

Comentario: Sólo 2 de 88 estudiantes de niveles similares a $3^{\circ}$ de BUP, COU y un curso de formación inicial para profesores contestaron de forma correcta. Un $15 \%$ indicó que la trayectoria sería a lo largo de la línea recta que una las dos cargas. La mayoría (alrededor del 75\%) indica que la trayectoria será la de las líneas de campo. Estos resultados se pueden interpretar pensando que los estudiantes responden, buscando una solución rápida y segura, con una idea intuitiva de sentido común que relaciona fuerza y movimiento (el movimiento sigue la dirección de la fuerza). Así mismo, se observa la misma confusión entre campo y fuerza ya comentada en las preguntas anteriores.

2.5. La figura adjunta representa un corte perpendicular en sentido longitudinal a través de una piezas metálicas. A y C están cargadas, B es neutra. No existen corrientes y el sistema está en estado estacionario. En la figura se han dibujado una serie de líneas de campo eléctrico. Encontrar todos los errores de la figura y explicar por qué algunas de las líneas de campo no pueden dibujarse de esa manera.

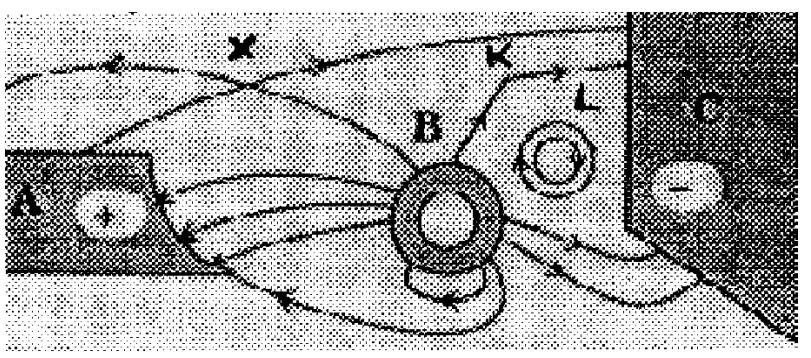

Referencia: Törnkvist, Pettersson y Tranströmer (1993)

Comentario: Esta cuestión ha sido contestada por 545 estudiantes de $2^{\circ}$ de universidad como parte de su examen de una asignatura de electricidad y magnetismo. Se analizaron detalladamente los ítems referentes a los errores L (líneas en círculo), K (líneas en ángulo) y X (líneas que se cruzan). El resultado más alarmante fue que el $85 \%$ de los estudiantes no detectaba el error denominado X. Así mismo, sólo un $4 \%$ explicaba de 
forma correcta que las líneas de campo no pueden formar un ángulo como el dibujado en K. Otros resultados son que el $29 \%$ de las explicaciones del error L y el $49 \%$ de las explicaciones del error K son de tipo «ingenuo», es decir, que parecen basadas en la intuición más que en aplicaciones de lo que se ha enseñado en clase. Todo ello muestra que los estudiantes no dominan suficientemente los conceptos matemáticos de continuidad de una función y sus derivadas como para aplicarlos en un contexto no matemático y, por tanto, no utilizan argumentaciones basadas en estos conceptos matemáticos. Así mismo, los estudiantes tienden a tratar las líneas de campo como entidades aisladas en un campo euclidiano más que como un conjunto de curvas que representan una propiedad vectorial del espacio.

2.6. La figura muestra un bote metálico cargado negativamente, ¿qué sucederá al introducir un péndulo cargado positivamente dentro del mismo?

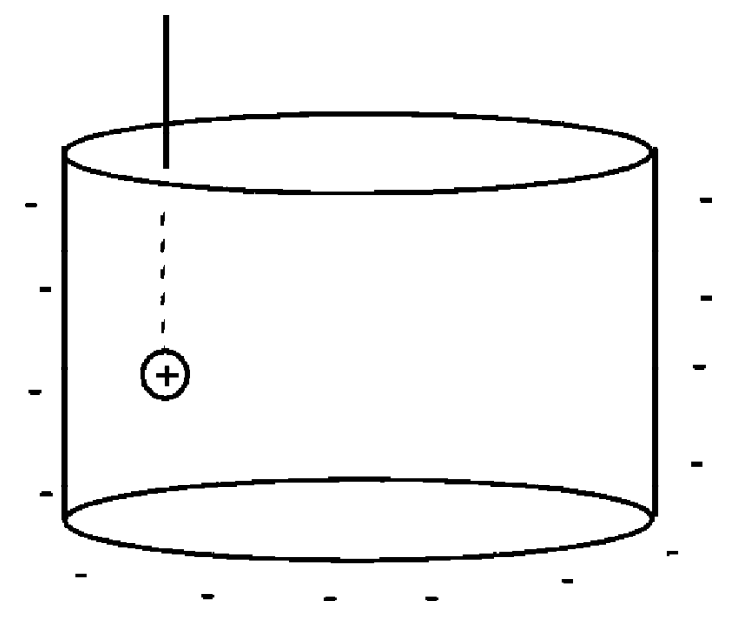

Referencia: Furió y Guisasola (1998b)

Comentario: La solución a la situación planteada requiere un análisis de la interacción eléctrica basada en el concepto de campo eléctrico y, consecuentemente, en la aplicación del teorema de Gauss. También cabe la posibilidad más complicada de aplicar la ley de Coulomb para elementos de superficie cargada del cilindro y posteriormente integrar en toda la superficie. Esta posibilidad no ha aparecido en ninguna de las respuestas analizadas. La cuestión ha sido contestada por 245 estudiantes de niveles de bachillerato y $1^{\circ}$ y $3^{\circ}$ de ciencias físicas. El porcentaje de respuestas correctas es muy bajo incluso en niveles universitarios $\left(0 \%\right.$ en $3^{\circ}$ BUP y COU, $10 \%$ en $1^{\circ}$ de físicas y $14 \%$ en $3^{\circ}$ de físicas). La respuesta errónea mayoritaria consiste en una aplicación de la ley de Coulomb, donde se considera cada lado de la pared del cilindro como una carga puntual y se aplica la proporcionalidad inversa entre distancia y fuerza. De acuerdo con los resultados, es claro que los estudiantes (incluidos los universitarios) no utilizan de forma signi- ficativa el concepto de campo eléctrico en un contexto electrostático. Así mismo, este resultado viene a coincidir con los resultados de otras cuestiones anteriores en el sentido de que este concepto carece de significación para una mayoría de estudiantes que ya han cursados varios años de teoría eléctrica.

2.7. Una esfera de material aislante de radio $R$ tiene una densidad de carga $r$ uniforme. La distribución de la carga fuera de la esfera es desconocida. Se quiere calcular el campo electrostático en el punto $M$ que está en el interior de la esfera, es decir, a una distancia del centro $r<R$. ¿Se puede realizar el cálculo pedido sin conocer la distribución de la carga fuera de la esfera?

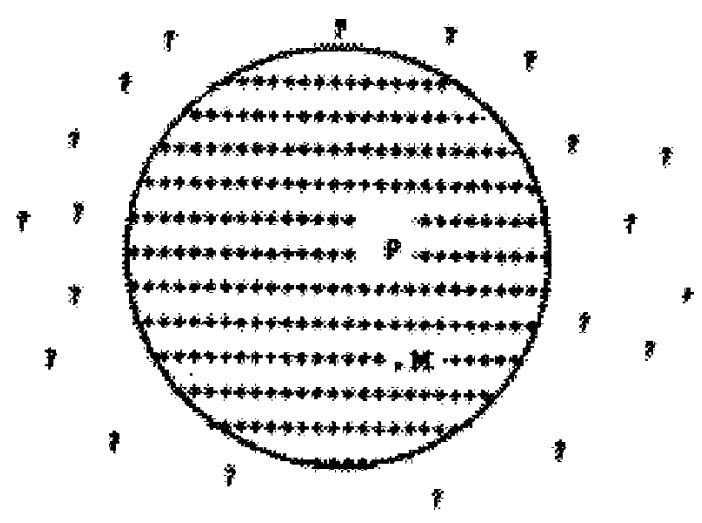

Referencia: Viennot y Rainson (1992)

Comentario: La cuestión ha sido contestada por dos muestras de estudiantes de $2^{\circ}$ curso de las universidades de París VII $(\mathrm{N}=108)$ y Setif en Argelia $(\mathrm{N}=29)$. La respuesta correcta es muy minoritaria (15\% en París y $14 \%$ en Setif) e implica tener en cuenta que la carga externa influye en la distribución de la carga en la esfera $\mathrm{y}$, por tanto, en el cálculo de la intensidad de campo en el punto $M$, por ejemplo, al aplicar el teorema de Gauss. La mayoría de las respuesta (83\% en París y $86 \%$ en Setif) indican erróneamente que se puede calcular el campo sin tener en cuenta la distribución de carga exterior. Para justificar esta respuesta argumentan que al aplicar el teorema de Gauss sólo es necesario saber la carga interior a la superficie gaussiana, sin darse cuenta de que la distribución de ésta vendrá condicionada por la carga exterior. Así pues, la mayoría de los estudiantes considera que la única causa del campo producido en $M$ es la carga interior de la esfera y la condición de simetría es necesaria para aplicar el teorema de Gauss. Esto estaría en contradicción con el principio de superposición de campos eléctricos que indica que el campo en un punto es la suma de los campos producidos por cada una de las cargas presentes como si cada carga estuviera sola. Este principio es básico y ampliamente utilizado por los estudiantes para calcular campos eléctricos producidos por sistemas de cargas. Sin embargo, en el análisis de esta cuestión el principio es implícitamente negado y no 
ayuda a los estudiantes en su respuesta, lo que sugiere un aprendizaje no significativo del principio.

Una minoría de respuestas erróneas argumenta que no se tiene que tener en cuenta las cargas externas, ya que la esfera es de material aislante. Aquí aparecen otra vez las dificultades de los estudiantes para interpretar fenómenos eléctricos en medios materiales.

2.8. Sea un cuerpo conductor $C$ y una carga puntual $q$ situada fuera del conductor (como se ve en la figura). En un punto $P$ situado en la superficie del conductor hay una densidad superficial de carga $s$ y el vector $n$ se define en el punto $P$ y dirigido hacia fuera. Queremos calcular el campo eléctrico $E(M)$ en el punto $M$ fuera del conductor y muy próximo a $P$.

Un estudiante sugiere la siguiente expresión $\mathrm{E}(\mathrm{M})=\mathrm{s} / \mathrm{e}_{0}$ + Eq, donde Eq, es el campo debido a la carga puntual $q$.

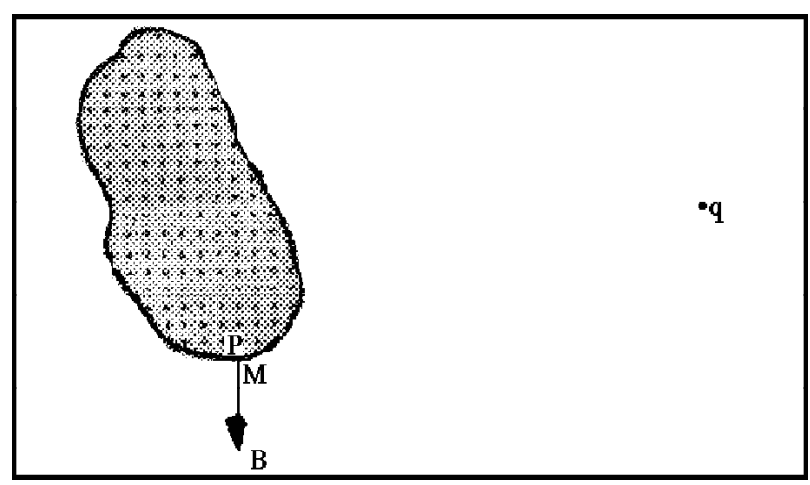

¿Qué piensas? La expresión es:

$\begin{array}{lcll}\text { a) Correcta } & \text { O Sí O No } & \begin{array}{l}\text { ¿Puedes explicar por } \\ \text { qué? }\end{array} \\ \text { b) Incorrecta } & \text { O Sí O No } & \begin{array}{l}\text { ¿Puedes decir por qué } \\ \text { y dar la respuesta } \\ \text { correcta? }\end{array} \\ \text { c) Ambigua } & \text { O Sí O No } & \begin{array}{l}\text { Explica por qué y da } \\ \text { una fórmula no } \\ \text { ambigua. }\end{array}\end{array}$

Referencia: Rainson, Tranströner y Viennot (1994), Viennot (1996)

Comentario: La cuestión fue contestada por 141 estudiantes de un nivel similar a $1^{\circ}$ y $2^{\circ}$ curso de carrera de ciencias o ingeniería. Alrededor de $1 / 3$ de los estudiantes encuestados indica que la fórmula es correcta, es decir, realizan una aplicación incorrecta del principio de superposición, ya que indican que el campo creado por el conductor en el punto $\mathrm{M}$ viene dado por $s / e_{0} \mathrm{y}$ que hay que sumarle el campo debido a la carga puntual $q$. Es más, 1/4 de los estudiantes que dicen que la expresión es ambigua profundizan en el argumento anterior al indicar que la presencia de la carga puntual $q$ produce una nueva distribución de la densidad de carga en el conductor y que, por tanto, la expresión debería ser $s$ ' $/ e_{0}$ + Eq. Se constata que la mayoría de los estudiantes es de la opinión que la expresión $s / e_{0}$ es debida exclusivamente a la carga del conductor y, por ello, tienen que sumar el campo de la carga puntual. Como mínimo el $75 \%$ de los estudiantes de cada curso muestra claramente que no entiende la situación planteada, y no analiza que en la expresión $s / e_{0}$ se incluye cualquier distribución externa de carga (en un punto muy próximo a la superficie del conductor) y, por tanto, no se puede sumar Eq a esta expresión. En este caso se observa cómo la comprensión incompleta de fórmulas correctas (en su contexto) puede suponer un obstáculo para la aplicación significativa de la teoría.

2.9. Una carga puntual está situada en el exterior de un cuerpo de material aislante. ¿Crea esta carga un campo en un punto $M$ situado en el interior del cuerpo?

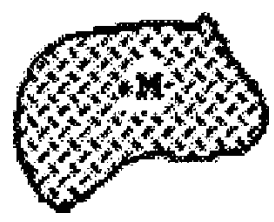

$+4$

Referencia: Viennot y Rainson (1992), Viennot (1996)

Comentario: La cuestión ha sido contestada por estudiantes de $1^{\circ}$ y $2^{\circ}$ curso de universidad $(\mathrm{N}=153)$. El porcentaje de respuestas correctas que razonan en base a la polarización del dieléctrico y, por tanto, a la existencia de un campo en $\mathrm{M}$ es muy pequeño (0\% a $8 \%$ ). Dentro de las respuesta erróneas, existe un porcentaje significativo (del $44 \%$ al $20 \%$ según el curso) que indica que no existe campo en $\mathrm{M}$ debido a que está en un aislante. Así mismo, otro porcentaje importante de respuestas erróneas (del 25\% al 14\% según el curso) liga la no existencia de campo en $\mathrm{M}$ a que las cargas en un aislante no se pueden mover. Así pues, parece que un alto porcentaje de estudiantes relaciona la no-movilidad de las cargas o el carácter aislante del material con la existencia de campo eléctrico. El resultado sugiere que los estudiantes necesitan imaginar un efecto (movimiento de cargas) para aceptar una causa. Este tipo de razonamiento también se observa en otros campos de la física, como la mecánica.

\section{DIFICULTADES EN EL APRENDIZAJE DEL CONCEPTO DE POTENCIAL ELECTRICO Y EN LA CAUSALIDAD DEL MOVIMIENTO DE LAS CARGAS}

3.1. Determinar la diferencia de potencial entre dos puntos de un circuito que contiene una batería si existe un interruptor abierto entre ambos puntos. 


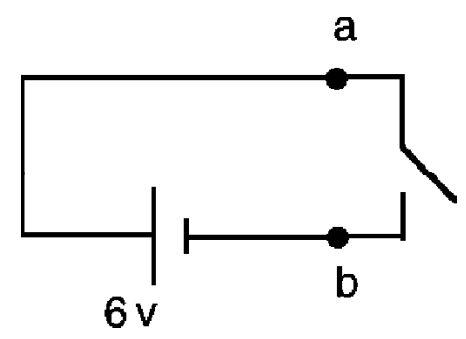

Referencia: Steinberg (1992)

Comentario: La gran mayoría de los estudiantes contesta que la diferencia de potencial entre $a$ y $b$ es cero. Justifica la respuesta indicando que «no hay corriente» y que el resultado de aplicar la ley de Ohm implica que la diferencia de potencial entre los dos puntos es cero. El autor sugiere que este resultado puede interpretarse considerando que los estudiantes piensan en la diferencia de potencial como una consecuencia del flujo de cargas más que como su causa. Es decir, no están pensando en la diferencia de potencial como un agente causal asociado con la acumulación de cargas. Así mismo, el autor opina que el pobre resultado obtenido en el aprendizaje del concepto de potencial puede ser atribuido a una introducción del concepto puramente operativa como, por ejemplo, el trabajo desarrollado al llevar la unidad de campo de un punto $\mathrm{A}$ a otro $\mathrm{B}$, o bien la fórmula $d V=$
$E d s$. Estas definiciones tienen significado para los expertos, ya que relacionan valores de diferencia de potencial con valores de otra magnitud (el campo eléctrico o el trabajo) para un eficiente cálculo. Pero son poco significativas para los estudiantes porque sólo definen el aspecto cuantitativo del potencial y, frecuentemente, sólo representan una fría fórmula. Así mismo, en este trabajo se realiza un estudio del desarrollo histórico del concepto de potencial para justificar la hipótesis de que el problema del aprendizaje del concepto de potencial radica en que los libros de texto realizan una presentación que es el resultado de un proceso de selección histórica dominado por el instrumentalismo matemático (Stocklmayer y Treagust, 1994). Se utiliza el concepto de forma puramente operativa y se pide a los estudiantes que realicen un salto a través de la matemática formal. Es aquí donde muchos no logran dar el salto. Así mismo, frecuentemente en la enseñanza habitual y en los libros de texto, se presenta una visión aproblemática que no explica, por ejemplo, por qué es necesario introducir el concepto de potencial eléctrico (Furió y Guisasola, 1997).

3.2. En la figura tenemos representadas dos esferas metálicas cargadas en tres situaciones diferentes, donde A tiene la mitad de diámetro que B. Se conectan entre sí mediante un hilo conductor. Explica en cada caso si habría movimiento de cargas y explica cómo quedaría la carga de cada esfera (dibuja lo que corresponde al estado final).
B

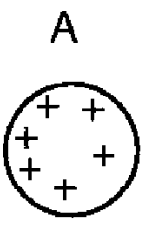

A

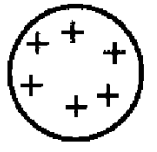

A

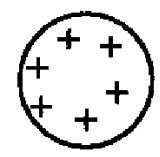

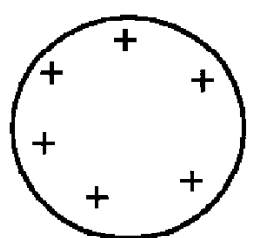

$\mathrm{B}$

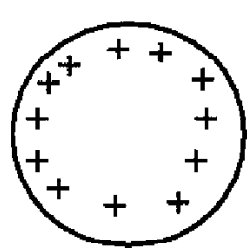

$\mathrm{B}$

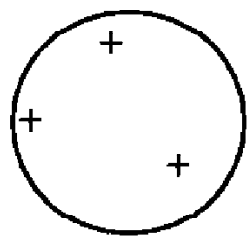

$\square$ sí

no

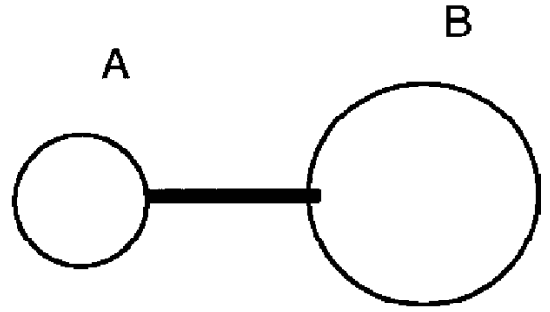

B

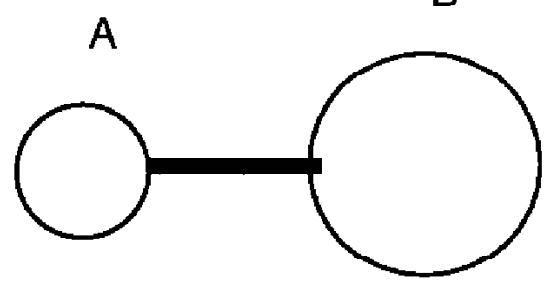

$\mathrm{B}$

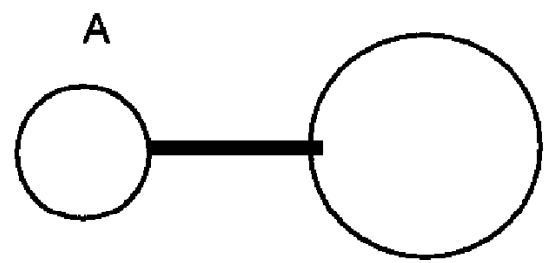


Referencia: Furió y Guisasola (1998c), Guisasola (1996)

Comentario: La cuestión planteada exige dar una razón sobre la causa del movimiento de las cargas. Fue contestada por una muestra de 247 estudiantes de bachillerato y $1^{\circ}$ y $3^{\circ}$ de ciencias físicas. La mayoría de los estudiantes responde incorrectamente, basando sus respuestas en dos tipos de argumentos. El primero se refiere a que no es posible el movimiento de cargas, ya que cargas del mismo signo se repelen (alrededor del $50 \%$ de las respuestas en bachillerato, $32 \%$ en $1^{\circ}$ de físicas y $5 \%$ en $3^{\circ}$ de físicas). El segundo argumento se refiere a que las cargas se mueven hasta igualar la cantidad de cargas en ambas esferas (alrededor del $6 \%$ en cada uno de los niveles). Así mismo, las respuestas correctas se clasifican en dos categorías. La primera agrupa a aquéllas que atribuyen el movimiento de cargas a la existencia de una diferencia de potencial entre ambas esferas $(0 \%$ en bachillerato, $15 \%$ en $1^{\circ}$ de físicas y $28 \%$ en $3^{\circ}$ de físicas. La segunda agrupa las explicaciones que considera, como causa del movimiento de las cargas, una distribución igualitaria de la densidad de carga en ambas esferas, es decir, no sólo se tiene en cuenta la cantidad de cargas en cada esfera sino también el tamaño de las mismas (alrededor del $20 \%$ en bachillerato y $1^{\circ}$ de físicas y $17 \%$ en $3^{\circ}$ de físicas). Se observa un alto porcentaje de estudiantes que no contestan (alrededor de la cuarta parte). Esto, junto con los resultados expuestos, muestra las serias dificultades de los estudiantes para razonar sobre la causalidad del movimiento de las cargas. Así mismo, el trabajo muestra que la no-relación por parte de los estudiantes entre el concepto energético de potencial y el movimiento de las cargas es una consecuencia lógica de la carencia de significado de este concepto para los estudiantes. Esta conclusión vendría a converger con las conclusiones del trabajo anteriormente expuesto (Steimberg, 1992) y con los que comentaremos a continuación.

\section{3 .}

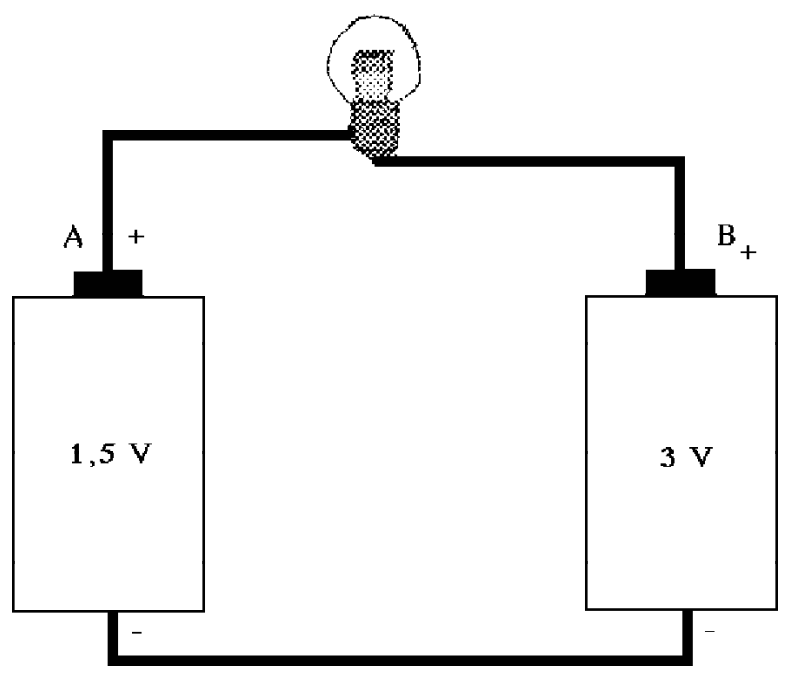

a) ¿Existe diferencia de potencial entre los puntos A y $\mathrm{B}$ ?
O Sí
O No
¿Por qué?

b) ¿Se encenderá la bombilla?
O Sí
O No
¿Por qué?

Referencia: Bensheguir y Closset (1996)

Comentario: Para la cuestión $a$, la respuesta «No» (incorrecta) es indicada por la mayoría de los estudiantes de bachillerato (del $43 \%$ al $70 \%$ para estudiantes de bachillerato) y por casi la mayoría de los estudiantes de $1^{\circ}$ y $2^{\circ}$ curso de universidad (47\%). El razonamiento que siguen para justificar la respuesta es que los dos polos conectados son positivos y, por tanto, no se crea diferencia de potencial. Así mismo, del análisis de la correlación entre las respuestas a las cuestiones $a$, y $b$, los autores muestran que para algunos estudiantes la diferencia de potencial entre los bornes de la pila y la circulación de corriente están en registros diferentes: la diferencia de potencial es considerada de forma estrictamente numérica como una característica de la pila y la corriente eléctrica se analiza a partir de consideraciones electrostáticas: atracción entre cargas, signo diferente de las cargas en los extremos.

Los autores del trabajo también se preguntan sobre la posibilidad de poder observar el razonamiento «electrostático» de los estudiantes en un contexto electrocinético sin que intervengan directamente los bornes de la batería. Es decir, los autores pretenden analizar la comprensión de los estudiantes sobre el potencial en cualquier punto del circuito. Aquí expondremos a continuación una de las cuestiones que los autores proponen:

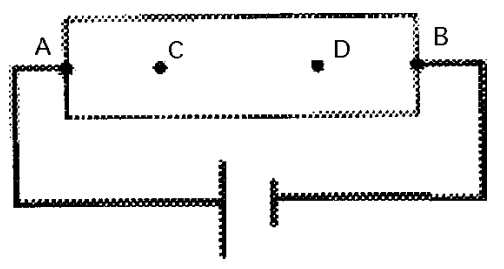

El dibujo muestra una resistencia cuyas extremidades A y B están conectadas con los bornes de la batería. Los puntos C y D están dentro de la resistencia. Los estudiantes deben contestar si existe diferencia de potencial entre los puntos A y B y los puntos C y D. Los resultados muestran que para los estudiantes el estatus de los puntos C y D es muy diferente del de los puntos A y B (la mayoría de los estudiantes de bachillerato y 1 de cada 3 estudiantes de universidad indican que no hay diferencia de potencial entre $\mathrm{C}$ y $\mathrm{D}$ ). Un número significativo de estudiantes sólo considera una diferencia de potencial entre aquellos puntos del circuito donde se percibe la diferencia de signos (en A y B se ve claramente) o de cantidad de cargas entre los puntos (explican que dentro de la resistencia no hay variación de la cantidad de 
carga). Los autores atribuyen estos resultados a que los estudiantes consideran la diferencia de potencial como «abstracto» y prefieren un concepto mucho más accesible como es el de carga eléctrica.

3.4. Consideremos un circuito como el del diagrama adjunto, donde el hilo conductor se ha dibujado deliberadamente grueso (con sección recta constante, el material tiene resistividad constante $g$ ).

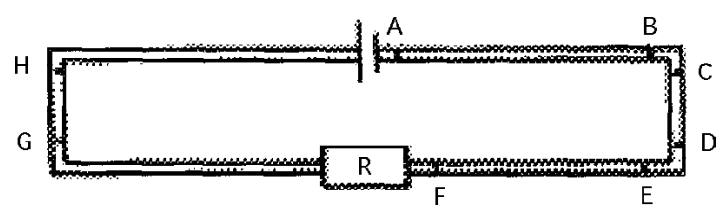

(El rectángulo identificado con una $\mathrm{R}$ representa una resistencia.)

$\mathrm{E}_{1}$ es el campo eléctrico en el interior del cable en el tramo AB.

E2 es el campo eléctrico en el interior del cable en el tramo CD.

E3 es el campo eléctrico en el interior del cable en el tramo EF.

E4 es el campo eléctrico en el interior del cable en el tramo $\mathrm{GH}$.

1. Dibujar los vectores $\mathrm{E}_{1}, \mathrm{E}_{2}, \mathrm{E}_{3} \mathrm{y} \mathrm{E}_{4}$. Justificar el dibujo (dirección y magnitud).

2a. ¿Cuáles son las fuentes de estos campos?

b. ¿Cómo puedes explicar las posibles variaciones de la dirección del campo de un tramo a otro?

c. ¿Has pensado alguna vez sobre este problema?

\section{Referencia: Rainson, Tranströmer y Viennot (1994)}

Comentario: La cuestión va dirigida a investigar hasta qué punto los estudiantes tienen una concepción integrada de los fenómenos eléctricos. Es decir, a ver de qué modo relacionan los estudiantes un concepto de electrostática como el campo eléctrico y los circuitos eléctricos. La respuesta correcta a la cuestión es que la dirección del campo eléctrico es la misma que la de la corriente $(\mathrm{E}=\mathrm{s} \mathrm{J}$, donde $\mathrm{J}$ es la densidad de corriente $\mathrm{y}$ $\mathrm{s}=1 / \mathrm{g}$ es la conductividad), las fuentes del campo eléctrico son todas las cargas del cable y la distribución cargas en la superficie del hilo son las responsables del cambio de dirección del campo (Chabay y Sherwood, 1995). La cuestión fue respondida por una muestra de 332 estudiantes universitarios de dos cursos. La gran mayoría ( $79 \%$ de $1^{\circ}$ y $82 \%$ de $2^{\circ}$ ) indica que las direcciones del campo y la corriente son paralelas. Sin embargo, a la hora de explicar las fuentes del campo se produce una gran diversidad de respuestas, las más significativas son: a) «La pila», o «el voltaje de la pila», o «los bornes de la pila» $\left(23 \%\right.$ de $1^{\circ}$ y $52 \%$ de $\left.2^{\circ}\right)$.

b) «El voltaje» sin referirse a la pila, o la energía potencial, $\mathrm{o}\left\langle\mathrm{E}=\mathrm{U} / \mathrm{d} \gg, \mathrm{o} \gg \mathrm{E}=-\operatorname{grad} \mathrm{V} \gg\left(15 \%\right.\right.$ de $1^{\circ}$ y $10 \%$ de $2^{\circ}$ ).

c) «La corriente» $\mathrm{O}$ «los electrones» del cable $\left(17 \%\right.$ de $1^{\circ}$ y $18 \%$ de $2^{\circ}$ ).

En cuanto al cambio de dirección del campo, los estudiantes lo atribuyen principalmente a dos razones:

d) «La forma del hilo» $\left(22 \%\right.$ de $1^{\circ}$ y $27 \%$ de $\left.2^{\circ}\right)$.

e) «La dirección de la corriente mueve los electrones» ( $13 \%$ de $1^{\circ}$ y $17 \%$ de $2^{\circ}$ ).

Así mismo, sólo el 6\% de los estudiantes indica que habían pensado antes sobre estas cuestiones.

Los resultados llevan a los autores a afirmar que para estos estudiantes los conceptos trabajados en electrostática y los circuitos eléctricos son dos temas no relacionados e independientes. A veces la corriente es indicada como la causa del campo eléctrico (respuesta $c$ ) y frecuentemente el análisis de la causa de la corriente es muy pobre. Una causa global como la pila o la fórmula $\mathrm{E}=\mathrm{U} / \mathrm{d}$, es suficiente. Quizás, la conclusión más importante sea que no existe un cuestionamiento espontáneo del cambio de la dirección del campo por parte de los estudiantes; es admitido como «normal». Parece que la concepción del campo eléctrico está fuertemente condicionada por la naturaleza del fenómeno: el campo eléctrico no es el mismo concepto cuando las cargas se están moviendo en el hilo que cuando están quietas.

3.5. El circuito adjunto contiene: una pila, un interruptor $\mathrm{S}$, un condensador con placas $\mathrm{A}$ y $\mathrm{B}$, una resistencia y dos amperímetos 1 y 2. (El condensador y la resistencia tienen valores grandes $\mathrm{R}>10^{6} \mathrm{~W}, \mathrm{C}>10 \mathrm{mF}$. Los valores exactos no son importantes.)

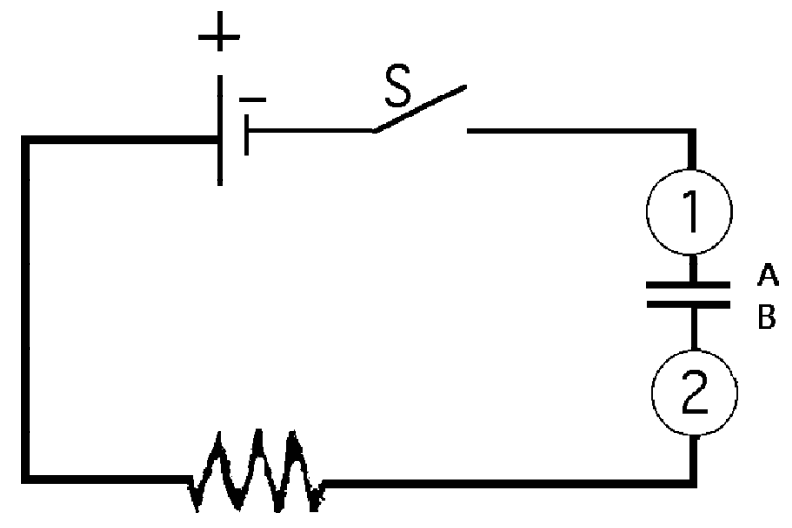

$\mathrm{El}$ interruptor $\mathrm{S}$ se cierra en $\mathrm{t}=0$. Describir de forma cualitativa: 
1.a) ¿Qué indicará el amperímetro 1 a lo largo del tiempo desde $t=0$ en adelante?

b) Explicar lo mismo para el amperímetro 2.

c) Si el amperímetro 1 indica en un momento dado $i_{1}$ y el amperímetro $2 i_{2}$, ¿cuál es la relación entre las dos? ( Es $i_{1}$ mayor, igual o menor que $i_{2}$.)

d) Cuando el interruptor S está abierto, las placas A y $\mathrm{B}$ del condensador no están cargadas. De el instante inicial en adelante:

- ¿Se acumularán cargas en la placa A? Si es así, ¿de qué signo (positivo o negativo)?

- Explicar lo mismo para la placa B.

2. De acuerdo con lo que has respondido en la cuestión anterior:

a) ¿Cuál es el comportamiento de la corriente en el amperímetro 1 a lo largo del tiempo? (Elige una de las respuestas, explícala utilizando términos como carga, corriente, fuerza, campo eléctrico, potencial, diferencia de potencial, voltaje.)

1) La corriente es cero. ¿Por qué?

2) La corriente es constante y distinta de cero. ¿Por qué?

3) La corriente aumenta hasta un valor máximo y luego permanece constante. ¿Por qué aumenta la corriente? ¿Por qué permanece constante?

4) La corriente aumenta de forma instantánea hasta un valor inicial y después decrece gradualmente hasta cero. ¿Por qué hay una corriente inicial? ¿Por qué decrece y se hace cero al final?

5) Otra posibilidad. Describirla y explicarla.

b) Explicar lo mismo para el amperímetro 2.

c) Las siguientes preguntas se refieren a la carga existen-

\section{REFERENCIAS BIBLIOGRÁFICAS}

ABIMBOLA, I. (1988). The problem of terminology in the study of student conceptions in science. Science Education, 72, pp. 175-184.

AUSUBEL, D.P. (1978). Educational Psychology. A cognitive view. Nueva York: Holt, Rineheart and Winston, Inc.

BENSHEGUIR, A. y CLOSSET, J.L. ( 1996). The electrostaticselectrokinetics transition: historical and educational difficulties. te en las placas A y B desde el tiempo cero en adelante.

1) No se acumulará carga. ¿Por qué?

2) Se aculumará carga. ¿Por qué se acumula? ¿Cuál es la fuente de estas cargas? ¿Si en un momento dado, la placa A tiene una carga qA y la placa B, una carga qB, cuál es la relación entre ambas (magnitud y signo)?

Referencia: Eylon y Ganiel (1990)

Comentario: las respuestas a las cuestiones fueron desarrolladas por 92 estudiantes de un curso similar al COU. La primera cuestión es contestada correctamente por el $80 \%$ de los estudiantes. Sin embargo, cuando en la cuestión 2 tienen que explicar los procesos que llevan a esos resultados, aparece una mayoría de respuestas incorrectas. Así, en la cuestión 2a y 2 b destacan aquéllas que se basan en considerar el condensador como un cortocircuito del paso de corriente $(30 \%)$. El $30 \%$ indica que $i_{1}=0$ e $i_{2}$ no es cero, basándose en un pensamiento secuencial que explica que todo sucede como si la corriente dejase la pila con un caudal inicial independientemente del circuito y después lo recorrerá dependiendo de los «obstáculos» que encuentre a su paso. En este caso, los estudiantes consideran que la corriente sí sale del polo positivo ( $i_{2}$ no es cero) pero que no llega al polo negativo porque lo impide el condensador $\left(i_{1}=0\right)$. Muy pocos estudiantes hacen referencia al campo eléctrico. La mayoría utiliza las palabras voltaje o diferencia de potencial pero de una manera vaga e imprecisa. En la pregunta $2 c$ es revelador que el $63 \%$ indique erróneamente, como única fuente de las cargas que se colocan en las placas, la pila, sin tener en cuenta los cables y el condensador. Los resultados se pueden interpretar pensando que los estudiantes no relacionan lo estudiado en electrostática y, en concreto, el concepto de diferencia de potencial con lo que sucede en el circuito. Así, el concepto de potencial que utilizan en las explicaciones no es preciso en lenguaje científico y, a la hora de interpretar lo que sucede, improvisan mecanismos en base a la intuición. Estas carencias impiden que los estudiantes interpreten el circuito como un sistema global. 
CHABAY, R. y SHERWOOD, B. (1995). Electric \& Magnetic interactions. Nueva York: John Wiley \& Sons, Inc.

CLOSSET, J.L. (1983). Le raisonament sequentiel en electrocinetique. Tesis doctoral. Université de Paris VII, Laboratoire de Didactique de la Physique dans l'Ensegnement Supérieur.

DRIVER, R. (1989). Students' conceptions and the learning of science. International Journal of Science Education, 11(5), pp. 481-490.

DUPIN, J. y JOSHUA, S. (1986). L'electrocinetique du College a l'Université: évolution de representations des élèves et impact de l'enseignement sur ces évolutions des representations. Bulletin de l'Union des Physiciens, 683, pp. 779-780.

EYLON, B.S. y GANIEL, U. (1990). Macro-micro relationships: the missing link between electrostatics and electrodynamics in students' reasoning. International Journal of Science Education, 12(1), pp. 79-94.

FURIÓ, C. (1996). Las concepciones alternativas del alumnado en ciencias: dos décadas de investigación. Resultados y tendencias. Alambique, 7, pp. 7-17.

FURIÓ, C. y GUISASOLA, J. (1993). ¿Puede ayudar la historia de la ciencia a entender por qué los estudiantes no comprenden los conceptos de carga y potencial eléctrico? Revista Española de Física, 7(3), pp. 46-50.

FURIÓ, C. y GUISASOLA, J. (1997). Deficiencias epistemológicas en la enseñanza habitual de los conceptos de campo y potencial eléctrico. Enseñanza de las Ciencias, $15(2)$, pp. 259-271.

FURIÓ, C. y GUISASOLA, J. (1998a). Dificultades de aprendizaje de los conceptos de carga y campo eléctrico en estudiantes de bachillerato y de universidad. Enseñanza de las Ciencias, 16(1), pp. 131-146.

FURIÓ, C. y GUISASOLA, J. (1998b). Difficulties on learning the concept of electric field. Science Education, 82(4).

FURIÓ, C. y GUISASOLA, J. (1998c). Construcción del concepto de potencial eléctrico mediante el aprendizaje por investigación. Revista de Enseñanza de la Física. (En prensa.)

GALILI, I. (1995). Mechanics background influences students' conceptions in electromagnetism. International Journal of Science Education, 17(3), pp. 371-387.

GIANNETTO, E., TARSITANI, C. y VICENTINI MISSONI, M. (1992). The relations between Epistemology, History of Science and Science Teaching from the point of view of the research on mental representations. Proceedings of the Second International. Conference on History and Philosophy of Science in Science Education, pp. 359-374. Toronto, Canadá.

GIL, D. y CARRASCOSA, J. (1985). Science learning as a conceptual and methodological change. European Journal of Science education, 5, pp. 70-81.

GUISASOLA, J. (1996). Análisis crítico de la enseñanza de la electrostática en el bachillerato y propuesta alternativa de orientación constructivista. Tesis doctoral. Departamento de Física Aplicada I de la Universidad del País VascoEuskal Herriko Unibertsitatea.

GUISASOLA, J. y FURIÓ, C. (1994). Dificultades en el aprendizaje significativo de algunos conceptos de electrostática. Investigación en la Escuela, 23, pp. 103-114.
HIERREZUELO, J. (1986). Revisión bibliográfica sobre la enseñanza de la energía. Enseñanza de las Ciencias, 4(1), pp. 66-88.

MANRÍQUEZ, M.J., VARELA, P. y FAVIERES, A. (1989). Selección bibliográfica sobre esquemas alternativos de los estudiantes en electricidad. Enseñanza de las Ciencias, 7(3).

MOREIRA M.A. (1994). Diez años de la revista Enseñanza de la Ciencias: de una ilusión a una realidad. Enseñanza de la Ciencias, 12(2), pp. 147-153.

NARDI, R. y CARVHALO, A.M.P. (1990). A genese, a psicogenese e a aprendizagem do conceito de campo: subsídios para a construção do ensino desse conceito. Caderno Catarinense do Ensino de Física, 7, pp. 46-69.

PERALES, F.J. y NIEVAS, F. (1988). Nociones de los alumnos sobre conceptos de óptica geométrica. Enseñanza de la Ciencias, 6(1), pp. 86-89.

PFUNDT, H. y DUIT, R. (1994). Bibliography: Students' alternative frameworks and science education (4a. ed.) Kiel (Alemania): Institute for Science Education at the University of Kiel.

RAINSON, S., TRANSTRÖMER, G. y VIENNOT, L. (1994). Students' understanding of superposition of electric fields. American Journal of Physics, 62(11), pp. 1026-1032.

SHIPSTONE, D., RHONECK, C., JUNG, W., KARRQUIST, C., DUPIN, J., JHOSUA, S. y LICHT, P. (1988). A study of students' understanding of electricity in five European countries. International Journal of Science Education, 10(3), pp. 303-316.

SOLBES, J. y MARTÍN, J. (1991). Análisis de la introducción del concepto de campo. Revista Española de Física, 5(3), pp. 34-39.

STEINBERG, M.S. (1992). What is electric potencial? Connecting Alessandro Volta and contemporany students. Proceeding of the Second International Conference on the History and Philosophy of Science and Science Teaching, Vol. II, pp. 473-480. Kingston.

STOCKLMAYER, S. y TREAGUST, D.F. (1994). A historical analysis of electric current in textbooks: a century of influence on physics education. Science \& Education, 3, pp. 131-154.

TÖRNKVIST, S., PETTERSSON K.A. y TRANSTRÖMER, G. (1993). Confusion by representation: on student's comprehension of the electric field concept. American Journal of Physics, 61(4), pp. 335-338.

VIENNOT,L. (1985). Analyzing students' reasoning: tendencies in interpretation. American Journal of Physics, 53(5), pp. 432-436.

VIENNOT, L. (1996). Raisonner en physique. París: De Boeck Université.

VIENNOT, L. y RAINSON, S. (1992). Students' reasoning about the superposition of electric fields. International Journal of Science Education, 14(4), pp. 475-487.

WANDERSEE, J.H., MINTZES, J.J. y NOVAK, J.D. (1994). Research on alternative conceptions in Science. Handbook of Research on Science Teaching and Learning. Nueva York: McMillan Publishing Company. 